
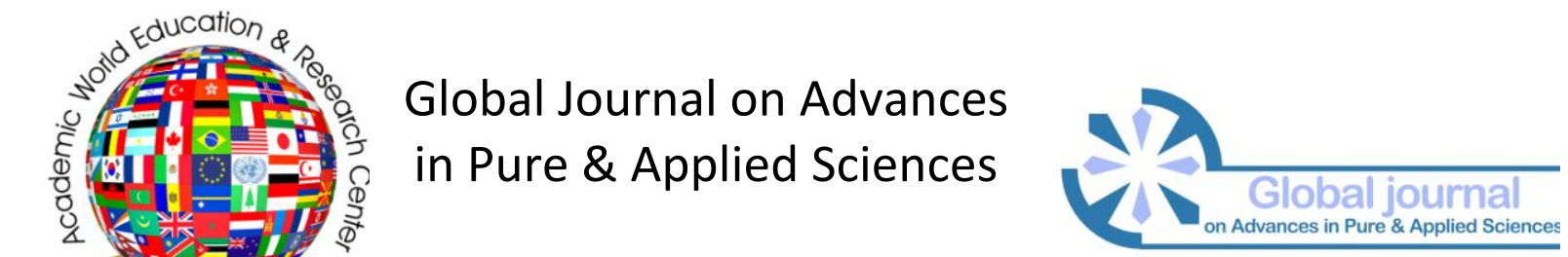

Issue 7 (2016) 65-72

Selected Paper of 2nd World Conference on Health Sciences (H-SCl 2015)

30 April-02 May 2015 Efes Sürmeli Hotel \& Convention Center - Izmir, Kuşadası, Turkey

\title{
Evaluation of important factors in accepts or reject of upper limb prosthesis in children
}

Meria Yazdani*, Rehabilitation School, Iran University of Medical Science, Iran, Islamic Republic Of.

Mohammad Kamali, Rehabilitation School, Iran University of Medical Science, Iran, Islamic Republic Of.

\section{Suggested Citation:}

Yazdani, M., \& Kamali, M. (2016). Evaluation of important factors in accepts or reject of upper limb prosthesis in children,

[Online]. 07, pp 65-72. Available from: $\underline{\text { www.propaas.eu }}$

Received November 02, 2014; revised January 11, 2015; accepted March 06, 2015.

Selection and peer review under responsibility of Prof. Dr. Fahrettin Sadikoglu, Near East University. C2016 Academic World Education \& Research Center. All rights reserved.

\begin{abstract}
Introduction: upper limb amputee Children usually uses prosthesis for better function. But studies show a high percentage of rejection of the prosthesis in this group. In clinical studies, there are not very different in function of children who use of prosthesis and those who do not. Answer to why children accept and use their prosthesis or reject it could help and improve the future design of the prosthesis and rehabilitation. The aim of this study was finding important criteria for children with below elbow amputation, their parents and therapists for acceptance and use of prosthetics or rejects it in children group. Method: This study was conducted by literature search of Science direct, Google Scholar, Cochran Library, MEDLINE and PubMed between 1966 and 2014 , that investigated effective factors in accept or reject of below elbow prosthesis in below elbow amputee children.For quality assessment of articles we rated each paper using the Downs and Black score ranges and Pedro scale. The two reviewers independently read and classified the articles by population, type of study and results. Results After initial evaluation and reviews, 14 articles were included in this study. Most studies were reported form and questionnaire and clinical observations. The general characteristics of the prosthesis, prosthesis control method, quality of life, family and the rehabilitation procedures were important factors were explored in these literature. Conclusion According to the studies, it is necessary to optimize the prosthesis according to the real children expectations and needs to improve their quality of life.There are controversial studies about performance and first time prosthesis in children but studies confirmed that Group therapy can increase the acceptance rate of
\end{abstract}

* ADDRESS FOR CORRESPONDENCE: Meria Yazdani, Rehabilitation School, Iran University of Medical Science, Iran, Islamic Republic Of. : merianil@yahoo.com 
Yazdani, M., \& Kamali, M. (2016). Evaluation of important factors in accepts or reject of upper limb prosthesis in children,

[Online]. 07, pp 65-72. Available from: www.propaas.eu

prosthesis. Training and finding ways for function without prosthesis along using it to communicate with the environment, increase motor control and sensory perception that could increase accept of prosthesis in children.

Keywords: upper limb prosthesis, children, amputation

\section{Introduction}

The main reason for not forming upper limb caused by congenital defects that usually appears with defects in other limbs. Other main Causes include trauma and tumors. Congenital below-elbow deficiency affect between 19.5 to 21.5 per 10,000 live births [1]. The exact number of upper limb amputee children in Iran is not available but in other countries, the data show that most amputation in children is below the elbow and in left side [2].

Children with such defects usually use of prosthesis to improve performance and prevent further complication but researches indicate that a high percentage of rejection of the prosthesis in this group that is approximately $10 \%$ to $49 \%$ [3]. Despite the efforts of designer and families, many children do not use of the prosthetic also there is not significantly different states between children who use of prosthesis and they are not used, One study in 489 children with upper limb amputations reported that approximately $34 \%$ of these children do not use of prosthesis or not accept it and the main reason for the rejection were dysfunction and no comfort of prosthesis [3]. In other studies functionality, simplicity, the appearance and social acceptance were important to considereation for proper design of children prosthesis $[1,2,4]$. Assessment that why children reject prosthesis and not used it could help to prosthesis designer or improve rehabilitation programs. The purpose of this Systmatic review is a descriptive analysis of the literatures with subject of children with below elbow amputation and assessement of children, parents and therapist recommendations in relation to accept or reject of upper limb prosthesis in these children. The main research question is What's the important factors in accept or reject of prosthesis by children, parents and therapists and Which eventually led to satisfaction and dissatisfaction of the prosthesis. Children are not the small adults [2], so their needs in assistive device and prosthesis must be considered as a distinct group.

\section{Material and Method}

In this study the published articles in field of "upper limb prostheses in children" from 1966 to 2014 were reviewed. The following databases was searched for articles: "Science direct; Google scholar; Cochrane Library; Medline; Pubmed and ISI web of science. "The keywords "upper limb prosthesis". "Upper limb extremity", "hand prosthesis", "upper limb deficiency" and "upper limb amputation" in combination with the words "children" and "infant" was used for research also, some of the refrences in articles were evaluated. The abstract and title of papers were evaluated based on selected criteria.

Selection criterias for inclusion to this study limited to researches in field of below elbow prosthesis in children who used passive, body power or myoelectric prosthesis. In these articles, important factors for accept or reject of prosthesis were prescribed by children, parents or therapists. Figure 1 , shows a diagram of study selection. In initial review of titles and abstracts, 160 articles was selected and at last 14 articles were selected after a review of the literature by inclusion criteria of research.

\section{Quality Assessment}

All the articles in terms of quality and methodology were rated separately by two investigators. The quality of the papers was evaluated based on "Down the Black tool" [6]. It is a valid test for assessing 
the quality of a systematic review and the PEDro tool was used to ranking papers [6, 7]. PEDro contains 11 questions with a maximum score of ten $[7,8]$ for grading, score of 5 or less, according to the article of Foley et al (2006), was considered as a poor quality of Randomized Controlled Trial [7] The final score for each article was with agreement of two authors. The relationship between test scores obtained by Pearson correlation test in Spss software version 17.

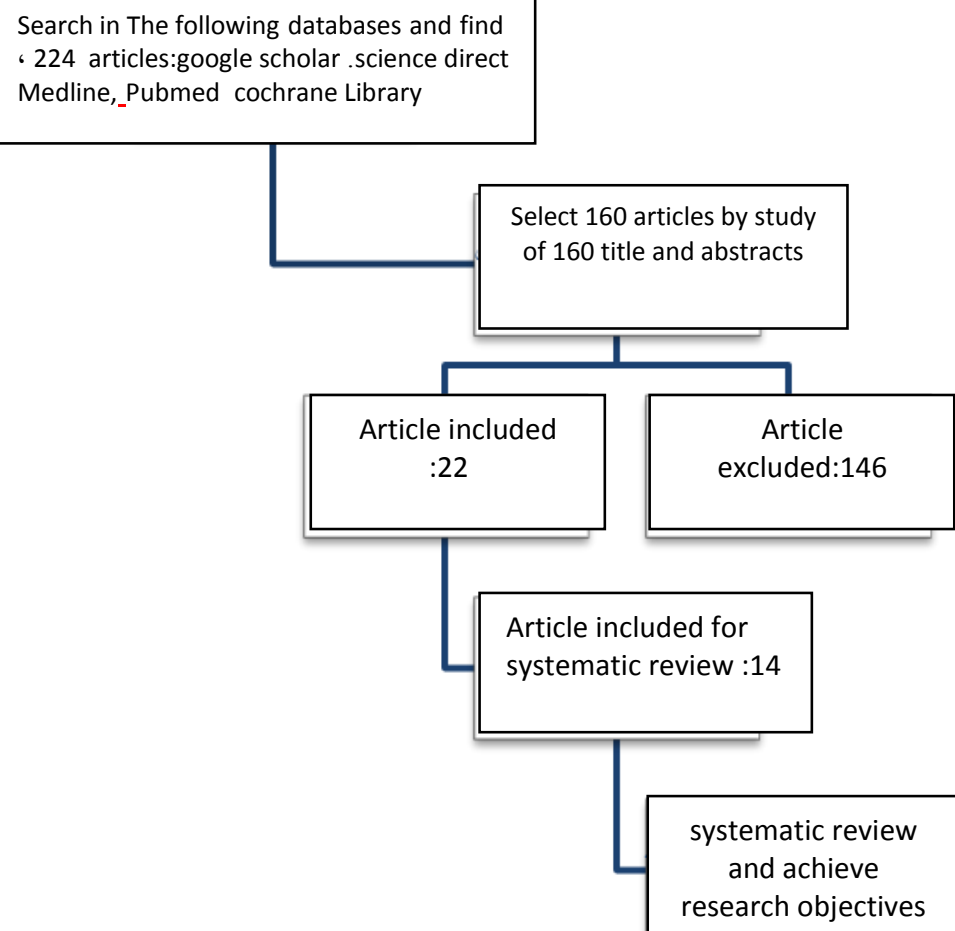

Figure 1. Flow chart of literature search for Evaluation of important factors in accept or reject of upper limb prosthesis in children

\section{Results}

This study is based on the study of 160 articles and discriptive analysis of 22 selective articles, that at last 14 articles included in research. Summary of descriptive analusis of included articles classified in Table 1. In table 2 also there are lists of the methods to evaluate the quality of the articles on the base of down and black check list that indicates, the reporting, external and internal validity, and total scores [6]. Ranking of the articles in this section were 3 to 8,1 to 3,2 to 5 and 2 to 5 , respectively for any items. 
Table 1. summary of included studies

Results: $\quad$ method $\quad$ Participant description $\quad$ Author, year

(ref.)

Reasons for rejection of prosthesis are, high confidence and ability of activity without prosthesis .cosmetic and structure of prosthesis were important factors for choose a prosthesis.

Early prosthetic fitting is valuable in accept of prosthetic.Cosmetic is important for children in specific age The reason of rejection of prosthesis was inability of prosthesis in function and structural and control problem

Uncomfortable harness and poor cosmetic were a close first and second for negative feature of arm prostheses

Most of the children with unilateral congenital deficiencies had received passive prostheses as their first prosthesesand body power as their second. Children with acquired defects usually had active prostheses.

Training is related to successful use of the prosthesis. The drop-out in preschool children is very low compared to adults. technical failure, breakdown, or fitting problems were reasons of temporary rejection

Children fit with a below elbow prosthesis at younger ages wore a prosthesis longer than children fitted at older ages. Type of prosthesis and area of living are effective in duration of using of prosthesis . voluntary opening body power prosthesis is not sufficient to greep for children

wearing a prosthesis can help doing only special sports or function. Although it is possible to fit a special sports device onto a prosthesis, it is (too) expensive Unilateral children amputees may choose multiple prostheses on the basis of function and the most functional and simple prosthesis in the long term design. Prostheses may help with social acceptance or as tools for specialized activities, but they do not appear to improve function or quality of life, functionality of prosthesis is not acceptable.

online focus group
interviews.Data
were analyzed using
the framework
approach
Case record and
questionner
Multi center cross
sectional study
Questionner ، and
open end questions

questionner

Interview and

Medical record

A specially designed questionnaire

retrospective chart review-interview

Experimental study - electronic dynamometer with force transducer A qualitative study using online focus group interviews

long-term follow-up questionnaire

A multicenter outcomes studyThe Unilateral Below-theElbow Test (UBET)
42 children of 8-12 year old y/o adolescents ; $13-16$ and 17-20 y/o, 17 parents, 19 healthcare professionals participated in study.

131 children with upper limb deficiencies were

Vasluian

2013

(ref.1)

Studied

489 children (2- 20 y/o ) with unilateral congenital below elbow amputation and parents

SCOTLAND, 1983

(ref.5)

Wagner

2007

(ref.3)

30 amputees ( 7 children) and their therapists

LeBlank

1985

(ref.8)

224 children, with unilateral congenital and aquaired

Kuyper

2001 deficiencies

(ref.9)

41 children with unilateral upper limb deficiency that use myoelectric prosthesis

Egermann 2009 (ref.10)

298 children with

Tokeshi

unilateral upper limb deficiency 2005

(ref.11)

37 limb deficient

SHAPERMAN

Children congenital, trans-

radial limb deficiencies.

1995 (ref.12)

42 children with

unilateral upper limb,

deficiency 17 parent and 19

normal subject

34 unilateral below-elbow amputees children

De Jong

2012

(ref.13)

Crandall

2002

(ref.14)

489 unilateral below-elbow

James amputees children

2006

(ref.15) 
Initial fitting before $1 \mathrm{y} / \mathrm{o}$ was related to use of a prosthesis for at least 4 years.Age at first fitting was not associated with satisfaction with the prosthesis, functional use of the prosthesis or motor skills.

The role of parents in the acceptance prosthesis is very important. Learning to operate prosthesis in children is better in the group.

Rejection within 3.5 years and after 13.5 years of prosthetic use is more, that relate to puberty. Fitting before the age of 2 years seems to reduce rejection rate.
The Satisfactory Inventory the Prosthetic Upper Extremity Functional Index Videotapes questionnaire

Cross-sectional study of a cohort questionnaire
20 children with congenital upper limb amputation Huizing 2010 between 6 to $21 \mathrm{y} / \mathrm{o}$

(ref.16)

12 Children with congenital, trans-radial limb deficiencies

Datta

1998

(ref.17)

22 children ( $0-18$ years) with a

Postema unilateral congenital arm 1999 defect and parents

Table 2. Quality assessment of included studies by Downs and Blak check list

\begin{tabular}{|c|c|c|c|c|c|c|}
\hline Total score & $\begin{array}{r}\text { selection } \\
\text { bias }\end{array}$ & $\begin{array}{r}\text { Internal } \\
\text { validity - bias }\end{array}$ & $\begin{array}{r}\text { External } \\
\text { validity }\end{array}$ & Reporting & author & refrence \\
\hline 16(fair) & 4 & 4 & 1 & 7 & Vasluian et al & 8 \\
\hline 20(good) & 5 & 5 & 2 & 8 & SCOTLAND et al & 4 \\
\hline 20(good) & 5 & 5 & 2 & 8 & Wagner et al & 7 \\
\hline 12(poor) & 3 & 3 & 1 & 5 & LeBlank et al & 99 \\
\hline 19(fair) & 5 & 4 & 2 & 8 & Kuyper et al & 10 \\
\hline 3(poor) & 3 & 3 & 1 & 6 & Egermann et al & 11 \\
\hline 21 (good) & 5 & 5 & 3 & 8 & Tokeshi et al & 12 \\
\hline 17(fair) & 4 & 4 & 2 & 7 & SHAPERMAN et al & 13 \\
\hline 21(good) & 5 & 5 & 3 & 8 & De Jong et al & 14 \\
\hline 20)good( & 5 & 5 & 2 & 8 & Crandal et al & 15 \\
\hline 21(good) & 5 & 5 & 3 & 8 & James et al & 16 \\
\hline 20(good) & 5 & 4 & 3 & 8 & HUIZING et al & 17 \\
\hline 10(good) & 2 & 2 & 1 & 5 & DATTA et al & 18 \\
\hline 20(good) & 5 & 5 & 3 & 7 & Postema et al & \\
\hline
\end{tabular}

Downs and Black score ranges were grouped into the following 4 quality levels: excellent (26 to 28 ), good (20 to 25), fair (15 to 19), and poor (less than 14).

\section{Discussion}

In this study after investigate of the study articles we found that function and control system, children condition, rehabilitation and family, early fitting of prosthesis and quality of life were total important factors discussed by children, parents or therapis that is discussed at below.

\section{Function and control system}

The performance and appearance of the prosthesis are important factors for accept. In this study, appearance of prosthesis even about function of prosthesis is very important factor for choosing a prosthesis by children and parents. [1,9,12,14,15], also the prosthesis can be reject by the poor performance, heavy weight, poor quality and lack of sensory feedback, battery discharge, motor problem and need to continiual maintenance $(1,10,16,19.19)$. The most of the children believe that the prosthesis, don,t help to normal function and the recreational activity $[3,4]$. 


\section{Children condition}

Age of early fitting, stump lenght and status and the physical and mental condition in children influence in accept of prosthesis. Child's high self-esteem and a sense of inability with the prosthesis cause rejection of prosthesis in some children.One study showed that children managed to function perfectly well without prostheses even are independence and doing good work [1]. However, certain activities such as lifting or some sports were not performed without prostheses by several young adolescent [8] children with long stamp usually are not a good candidate for use the prosthesis due to the high function and proprioceptive into the children with short stamp $[8,9,10]$.

\section{Rehabilitation and family}

One factor is a good fitting and alignment by expert prosthesists. A multidisciplinary rehabilitation team consisting of paediatric orthopaedic surgeons, prosthetists and occupational therapists accompanies the family during the rehabilitation program influence to the acceptance of prosthesis by children. Egermann (2009) concluded in this study that myoelectric prosthetic use training by an occupational therapist is related to successful use in children in 2 to 5 years [11]. Prosthetic use training is best in form of the games and before the child is tired. [1] also the role of parents in choice and acceptance of the prosthesis is as important as a child and they usually preffer the cosmetic passive prosthesis [1]. Also the role of parents and their guidance in the acceptance of body power and electronic prosthesis by children is very important $[17,18]$

\section{Early fitting of prosthesis}

Proper age for early fitting of Upper limb prostheses has been discussed in many studies and depend on Prescribed by a doctor or therapist. Parents believed that rapid administration of a prosthesis for the body image, balance and symmetry, acceptance and performance of children is important [8]. In some clinics early fitting is at the age of six months to support the sitting and crawling them [10]. The Scotland (1983) showed that the greatest acceptance of the prosthesis is under the age of two years and before enterance to school and $50 \%$ of children with unilateral amputation, use prosthesis after the age of two and $22 \%$ use earlier [5]. In Wagner study (2007) on 489 upper limb amputee, data from 110 subject who reject prosthesis use, showed that " $58 \%$ had been fitted befor age 1years of age and $18 \%$ had been fitted between 1 and 2 years of age" because of uncomfortability and not help to function. In another study Huizing (2010) concluded that prosthetic fittingt before 1 years age, leading to a longer use of prosthesis (more than four years), but does not improve motor behavior [16]. Some study showed that children in certain phases of the life (for example maturation) trend to reject of prosthesis $[5,9,18]$. Scotland $(1995)$ concluded that highest prosthesis drop-out rate was at the age of 13 years when the children became more sensitive to cosmetic appearance [5].

\section{Quality of life}

The primary expection and purpose of the amputee who use of Prosthesis is improve the quality of life [10]. For children with unilateral congenital below-elbow deficiency, prostheses appeared important for social integration more than functionality [1].

\section{Conclusion}

Prescription of upper limb prostheses for children requires special considerations [3]. This study shows that overall there is a need to optimization and improves the performance of the prosthesis for children and design of specific Prostheses and assistive device for daily work and recreation that could raise the percentage of acceptance of prosthesis in children. In this regard it is essential for prosthetic designers to Re-evaluate of the real role of prosthesis for improve the quality of life according to the 
expectations of the children and age. It should be noted that the Prostheses only will be accepted by children when it is a real assistance to help them and improve quality of life [20] The prosthesis acceptance by the children and family could raise by direct contact with the therapist or the same group as group therapy and training techniques and children education of alternative functional methods without the use of prosthetic $[1,3]$. Therapists should be aware of new prosthetic rehabilitation and technique to help this group [4,8]. Also design and use of quantitative and more accurate measurement tools to measure the performance of the prosthesis and the patients is necessery, specially for assessement of proper age for use of prosthesis in children. Early fitting of prosthesis, increse acceptance and tolerance of prosthesis and improve functionality $[5,19]$.

Overall this study showed that the structure and performance of the prosthesis and control system, quality of life ,prospect of family, time of prescription, and proper rehabilitation and training at the right time are important factors and ultimately led to the acceptance and satisfaction or rejection of the prosthesis. Optimization of the appearance, materials and control system and increased sensory feedback device in prosthesis, proper training along with similar groups and the use of a prosthesis with multiple performance can enhance the acceptance of the prosthesis. It should not be forgotten that for increasing sensory and motor skills in children it need to some training and strengthen the activities and alternative methods without the prosthesis. Children are not the small adults [2], so and prosthesis rehablitation for them must be considered as a distinct group.

\section{Refrences}

[1] Vasluian, E., de Jong, I. G., Janssen, W. G., Poelma, M. J., van Wijk, I., Reinders-Messelink, H. A., \& van der Sluis, C. K. (2013). Opinions of youngsters with congenital below-elbow deficiency, and those of their parents and professionals concerning prosthetic use and rehabilitation treatment.

[2] Krebs, D. E., Edelstein, J. E., \& Thornby, M. A. (1991). Prosthetic management of children with limb deficiencies.

(12), 920-934.

[3] Wagner, L. V., Bagley, A. M., \& James, M. A. (2007). Reasons for prosthetic rejection by children with unilateral congenital transverse forearm total deficiency.

(2), 51-54.

[4] Biddiss, E. A., \& Chau, T. T. (2007). Upper limb prosthesis use and abandonment: a survey of the last 25 years.

(3), 236-257.

[5] Scotland, T. R., \& Galway, H. R. (1983). A long-term review of children with congenital and acquired upper limb deficiency.

(3), 346-349.

[6] Downs, S. H., \& Black, N. (1998). The feasibility of creating a checklist for the assessment of the methodological quality both of randomised and non-randomised studies of health care interventions.

(6), 377-384.

[7] Foley, N. C., Bhogal, S. K., Teasell, R. W., Bureau, Y., \& Speechley, M. R. (2006). Estimates of quality and reliability with the physiotherapy evidence-based database scale to assess the methodology of randomized controlled trials of pharmacological and nonpharmacological interventions.

(6), 817-824.

[8] LeBlanc, M. A. (1985). Innovation and improvement of body-powered arm prostheses: A first step. , (1), 13-16.

[9] Kuyper, M. A., Breedijk, M., Mulders, A. H. M., Post, M. W. M., \& Prevo, A. J. H. (2001). Prosthetic management of children in The Netherlands with upper limb deficiencies.

(3), 228-234.

[10] Egermann, M., Kasten, P., \& Thomsen, M. (2009). Myoelectric hand prostheses in very young children.

(4), 1101-1105. 
[11] Shida-Tokeshi, J., Bagley, A., Molitor, F., Tomhave, W., Liberatore, J., Brasington, K., \& Montpetit, K. (2005). Predictors of continued prosthetic wear in children with upper extremity prostheses.

$$
\text { (4), 119-124. }
$$

[12] Shaperman, J., Leblanc, M., Setoguchi, Y., \& McNeal, D. R. (1995). Is body powered operation of upper limb prostheses feasible for young limb deficient children?

(3), 165-175.

[13] de Jong, I. G., Reinders-Messelink, H. A., Tates, K., Janssen, W. G., Poelma, M. J., van Wijk, I., \& van der Sluis, C. K. (2012). Activity and participation of children and adolescents with unilateral congenital below elbow deficiency: An online focus group study.

(10), 885892.

[14] Crandall, R. C., \& Tomhave, W. (2002). Pediatric unilateral below-elbow amputees: retrospective analysis of 34 patients given multiple prosthetic options.

(3), 380-383.

[15] James, M. A., Bagley, A. M., Brasington, K., Lutz, C., McConnell, S., \& Molitor, F. (2006). Impact of prostheses on function and quality of life for children with unilateral congenital below-the-elbow deficiency.

$$
\text { (11), 2356-2365. }
$$

[16] Huizing, K., Reinders-Messelink, H., Maathuis, C., Hadders-Algra, M., \& Van Der Sluis, C. K. (2010). Age at first prosthetic fitting and later functional outcome in children and young adults with unilateral congenital below-elbow deficiency: A cross-sectional study.

(2), 166-174.

[17] Datta, D., \& Ibbotson, V. (1998). Powered prosthetic hands in very young children.

$$
\text { (2), 150-154. }
$$

[18] Postema, K., Van der Donk, V., Van Limbeek, J., Rijken, R. A., \& Poelma, M. J. (1999). Prosthesis rejection in children with a unilateral congenital arm defect.

(3), 243-249.

[19] Philipson, L., \& Sörbye, R. (1981). Myoelectric elbow and hand prosthesis controlled by signals from 2 muscles only, in a 9 year old girl. , (1), 29-32.

[20] Meurs, M., Maathuis, C. G. B., Lucas, C., Hadders-Algra, M., \& Van der Sluis, C. K. (2006). Prescription of the first prosthesis and later use in children with congenital unilateral upper limb deficiency: A systematic review. $\quad, \quad(2), 165-173$.

[21] Lund, A. (1957). Observations on the very young upper extremity amputee.

22.

[22] Atlas of Amputations and Limb Deficienciesm,Surgical, Prosthetic, and Rehabilitation Principles, Douglas G. Smith, MD John W. Michael, MEd, CPO John H. Bowker, MD,Third Edition, 2004 